\title{
Anaphylaxis in Poland: the epidemiology and direct costs
}

\author{
Karina Jahnz-Rozyk ${ }^{1}$, Filip Raciborski², Andrzej M. Śliwczyński ${ }^{3,4}$, Anna Kłak ${ }^{5}$, Jarosław Pinkas ${ }^{6}$
}

${ }^{1}$ Department of Internal Diseases, Pneumonology, Allergology and Clinical Immunology, Military Institute of Medicine, Warsaw, Poland ${ }^{2}$ Department of the Prevention of Environmental Hazards and Allergology, Medical University of Warsaw, Warsaw, Poland

${ }^{3}$ Department of Quality of Medical Services, Procedures and Standards, Faculty of Health Sciences, Medical University of Lodz, Lodz, Poland

${ }^{4}$ Department of Analyses and Strategies, National Health Fund, Warsaw, Poland

${ }^{5}$ Department of Gerontology, Public Health, and Didactics, National Institute of Geriatrics, Rheumatology and Rehabilitation, Warsaw, Poland

${ }^{6}$ Department of Healthcare Organizations and Medical Certification, Centre of Postgraduate Medical Education, Warsaw, Poland

Adv Dermatol Allergol 2017; XXXIV (6): 573-579

DOI: https://doi.org/10.5114/pdia.2017.70361

\begin{abstract}
Introduction: Epidemiological data on anaphylaxis have been underestimated both in Poland and worldwide. Aim: To evaluate the prevalence of anaphylaxis in Poland, including a classification by gender, age and residential region. Material and methods: The data used in the analysis were derived from two sources, the National Health Fund records of healthcare services for 2008-2015 (official statistics) and a questionnaire-based survey conducted in 2015 on a sample of 305 allergists practicing in different regions of Poland.

Results: In 2015, 3144 people received treatment for anaphylactic shock (T78.0, T78.2, T80.5, T88.6) with an estimated prevalence rate of anaphylaxis of 8.2 per 100,000 ( 8.4 for females and 7.9 for males). The highest prevalence rate was found for women aged $50-54$ years $(14.5$ per 100,000). There was a very large difference in the prevalence of anaphylaxis between rural and urban areas (13.1 vs. 0.8 per 100,000). In 2015, the Polish NHF spent PLN 3.5 million (EUR 835,000) on the management of anaphylaxis. Of the allergists surveyed, $73 \%$ had been currently managing patients who had experienced anaphylactic shock. The most common causes of anaphylaxis included insect venom (41.4\%), food (29.8\%) and drugs (17.4\%).

Conclusions: A central anaphylaxis registry should be established in Poland. This is the only approach that would allow collecting a wide range of reliable information on the cases, management and consequences of anaphylaxis. Ongoing management of patients who have experienced anaphylactic shock should be improved.
\end{abstract}

Key words: anaphylaxis (anaphylactic shock), epidemiology, NHF (NFZ), costs, management.

\section{Introduction}

Anaphylaxis is the most dangerous form of an allergic reaction and may lead to death. Experts from the World Allergy Organization (WAO) define anaphylaxis as "a serious, life-threatening generalized or systemic hypersensitivity reaction" [1]. The American Academy of Allergy, Asthma \& Immunology (AAAAI) emphasizes that the criteria determining anaphylaxis are fulfilled when at least one of three conditions occurs within minutes to several hours following exposure to a trigger:

- acute onset of an illness with involvement of the skin and/or mucosa, as well as clinical manifestations expressed by at least one organ: respiratory system, hypotension, end-organ dysfunction;
- at least two of the following manifestations occurring rapidly after exposure to a potential allergen: involvement of the skin or mucosa, respiratory compromise, hypotension with accompanying signs and symptoms, or persistent gastrointestinal symptoms;

- a decrease in blood pressure after exposure to a known allergen for that patient - a decrease in blood pressure below the lower age-specific normal limit, or a greater than 30\% decrease in the baseline blood pressure [2, 3].

Anaphylactic shock is a hypersensitivity reaction that is quite life-threatening. The blood flow in tissues decreases with a resulting inadequate supply of oxygen and nutrients together with impaired waste product removal. This may occur both in subjects with a known allergy and those who have never experienced a hypersensitivity re-

Address for correspondence: Filip Raciborski PhD, Department of the Prevention of Environmental Hazards and Allergology, Medical University of Warsaw, 1 a Banacha St, 02-091 Warsaw, Poland, phone: +48 22599 20 39, e-mail: filip.raciborski@wum.edu.pl Received: 17.10.2016, accepted: 24.12.2016. 
action before. Because of cross-sensitivity, the onset of anaphylactic shock is sudden. Few patients seem to be aware that a secondary shock may occur a few hours after the first episode, usually within 4 to $12 \mathrm{~h}$. This is why each patient with the signs and symptoms of anaphylaxis should be admitted to hospital for 24-hour observation in case a secondary anaphylactic reaction occurs [4].

Epidemiological data on anaphylaxis have been underestimated both in Poland and worldwide. The World Allergy Organization (WAO) informs that globally the annual incidence of anaphylaxis ranges between 80 and 210 cases per million people. It has been estimated that approximately 0.05 to $2.0 \%$ of the world population have experienced anaphylaxis at least once at some point in their life [5, 6]. Biphasic anaphylactic reactions occur in $8-20 \%$ of cases while in $20 \%$ of cases the cause is unknown. According to WAO estimates, 1 to 5.5 people per million (1\%) die from anaphylaxis worldwide each year [5]. Based on clinical and administrative databases of hospitalized patients from different countries, the incidence and prevalence of anaphylaxis has been estimated to have increased seven-fold over the past 10-15 years. At the same time, the mortality rate from anaphylaxis has remained at the same level ( 0.35 to 1.06 cases per million people per year). Although death from anaphylaxis is infrequent, the risk of recurrence must be considered, as in the case of $26.5-54 \%$ of patients allergic to Hymenoptera venom [7].

In Europe, one in 300 people experiences anaphylaxis at least once at some point in their life [8]. In Germany, the incidence of anaphylaxis is 2-3 per 100,000 [9], in the USA, 40-50 per 100,000 per year [10] and in the UK, approximately 6-8 per 100,000 per year [11].

In adults, anaphylaxis is more frequent in women than in men. In those aged 13 to 56 years, the risk of severe anaphylaxis is higher in males than in females, while in children younger than 13 years and adults over 56 years of age there are no significant differences between genders [12].

Caution must be observed when interpreting data on the incidence and prevalence of anaphylaxis. It has been shown that anaphylaxis is often underdiagnosed, with the ICD-10 diagnosis codes, on which large epidemiological studies are based on, not precisely reflecting the current definition of anaphylaxis [13]. In the USA, anaphylaxis continues to be unrecognized in the Emergency Departments which results in its undertreatment in many cases as epinephrine is used in fewer than half of actually anaphylactic patients [14].

The most frequent triggers of anaphylaxis include insect stings and bites, foods, drugs and medical products. In over half of the cases in children, anaphylaxis is induced by food with only a small percentage of cases inducted by Hymenoptera venom or drugs. In adults, these proportions are reversed, and in the elderly the proportion of anaphylaxis triggered by drugs is higher [13]. In adults, the most frequent inducers of anaphylactic shock are, in decreasing order; drugs, insect venom, food and latex. In children, the order is reversed. The triggers are typically food, insect venom, drugs and only occasionally latex $[13,15]$.

Stinging insects of the order Hymenoptera have a venom that induces anaphylactic shock; typically bees, wasps, hornets and ants. The venom contains enzymes such as phospholipases and hyaluronidases, with other proteins that may trigger an IgE-dependent response [3]. Anaphylactic reactions to insect venom have a rapid onset, which is why they often lead to death from respiratory and circulatory failure [16].

In Poland, self-injectable epinephrine has been reimbursed since 2015. Hymenoptera venom immunotherapy (desensitization) is offered in Polish hospitals as a free healthcare service.

\section{Aim}

The aim of this study was to estimate the prevalence of anaphylaxis in Poland, including a classification by gender, age and regional residence (voivodeship), and to determine its most common triggers.

\section{Material and methods}

The study consisted of two independent stages: analysis of the data set sourced from the National Health Fund (NFZ), and analysis of a questionnaire-based survey conducted among Polish allergists.

Stage 1 of the study was performed on the data set sourced from the Polish National Health Fund (NFZ) for 2008-2015. The NFZ has a single-payer status in Poland. Its databases include information on all healthcare services financed from public funds provided in outpatient and inpatient settings (primary health care is financed on a flat-rate basis). Such data are collected by the NFZ for accounting purposes. In the present study, they were used to analyze the epidemiology of anaphylaxis in Poland. Secondary statistical analysis was employed.

For the purposes of the analysis, anaphylaxis (anaphylactic shock, anaphylactic reaction) was defined by the relevant ICD-10 diagnosis codes:

- T78.0 - anaphylactic reaction due to food;

- T78.2 - anaphylactic shock, unspecified;

- T80.5 - anaphylactic reaction due to serum;

- T88.6 - anaphylactic reaction following correct medicinal substance properly administered.

A person with a history of anaphylaxis was defined as a patient with at least one healthcare benefit (service) recorded in the NFZ database in the period covered by the study, with anaphylactic shock encoded by the relevant ICD-10 codes as the principal diagnosis or a co-existing disorder. The data used in the analysis were anonymized 
in compliance with the Polish Act on the Protection of Personal Data.

In some cases, healthcare professionals entered only the first three characters of the ICD-10 code in the medical records without providing a more detailed diagnosis, so some incidences of the following ICD-10 codes may have been included in the analysis:

- T78.8 - adverse effect, not otherwise classified;

- T80.8 - complications following infusion, transfusion or therapeutic injection;

- T88.8 - other complications of surgical and medical care, not elsewhere classified.

The prevalence rates for a given year were calculated by identifying the number of patients with a recorded ICD-10 code denoting anaphylaxis within the population of a given administrative region (voivodeship). The population estimates were obtained from the Central Statistical Office of Poland (GUS). If in a given year, a person received healthcare benefits (services) for anaphylaxis several times, they were treated as a single case of anaphylaxis for the purposes of the prevalence analysis.

Stage 2 of the study was a questionnaire-based survey conducted in 2015 in a group of 305 allergists practicing in Poland. Computer Assisted Telephone Interviews (CATI) were conducted by the professional journal Medycyna Praktyczna. The questionnaire consisted of 11 single choice or multiple choice questions. The data were entered into the database and subsequently the statistical analysis was performed.

In Poland in 2015, according to the Supreme Medical Council/Polish Chamber of Physicians and Dentists (NIL), there were 1250 certified physicians specializing in allergology. The structure of the surveyed group was generally in agreement with the NIL data on the percentages of all certified allergists practicing in particular administrative regions of Poland. A comparison of the two data sets is presented in Figure 1.
No Ethics Committee approval was required as both stages of the study were non-invasive and without the participation of patients.

\section{Statistical analysis}

Statistical analyses were performed using the following software: Excel, Statistica 10 and SAS EG 5.1. The analysis of the NFZ data did not include statistical significance tests and no confidence intervals were given as the NFZ data referred to the entire population in Poland. Descriptive statistics were used in the analysis of own data (CATI). Where justified, a 95\% confidence interval was constructed. For the causes of anaphylaxis, the confidence intervals were constructed based on the number of patients rather than physicians.

\section{Results}

\section{Secondary statistical analysis}

In Poland in 2008-2015, the number of patients per year who received healthcare benefits (services) for ICD10 diagnoses encoded T78, T80 or T88 increased over four-fold, from 27,100 to 111,400. In 2015, 66.2\% (73,800) of those patients had been diagnosed with another or an unspecified allergy (T78.4), a nearly eight-fold increase from 2008 (9,600).

The number of people per year who received healthcare benefits (services) because of anaphylaxis (T78.0, T78.2, T80.5, T88.6) in 2008-2013 ranged from 1963 in 2010 to 2344 in 2012. In 2014, the number increased to 2726 and in 2015 it was 3144. In 2015, a total of 10,131 patients received healthcare services related to anaphylaxis. This means that a patient from that group received healthcare services for anaphylaxis 3.2 times on average. The details are given in Figure 2.

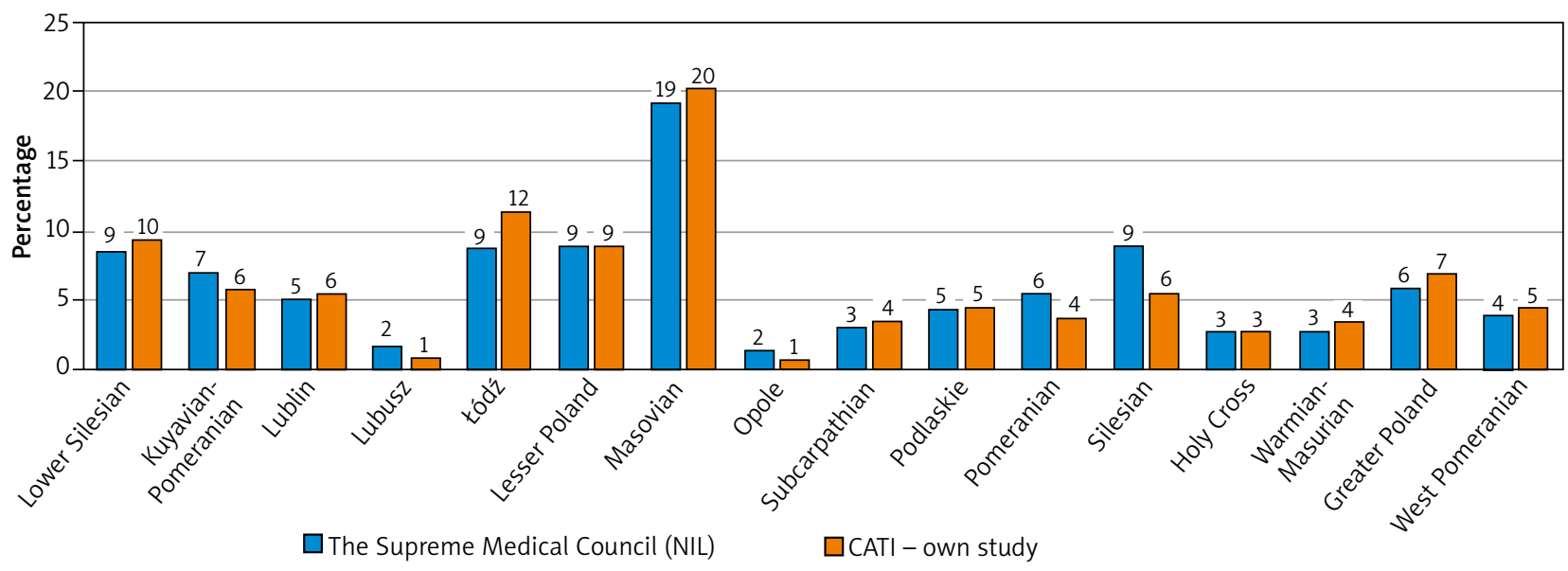

Figure 1. Percentages of all certified allergists practicing in particular administrative regions (voivodeships) of Poland. The NIL data vs. CATI data 


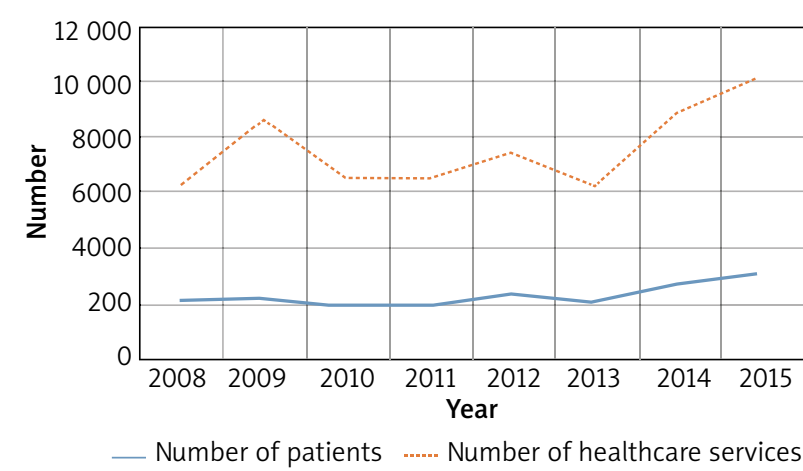

Source: Own analysis based on NFZ data.

Figure 2. The number of patients and healthcare services they received for anaphylaxis, 2008-2015

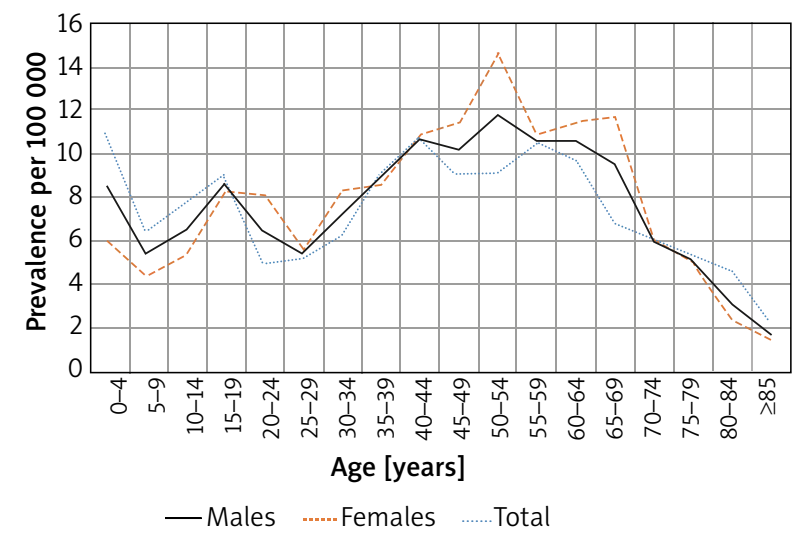

Source: Own estimate based on NFZ data.

Figure 3. Prevalence rates of anaphylaxis per 100,000 people, by age and gender (2015)

Source: Own estimate based on NFZ data.

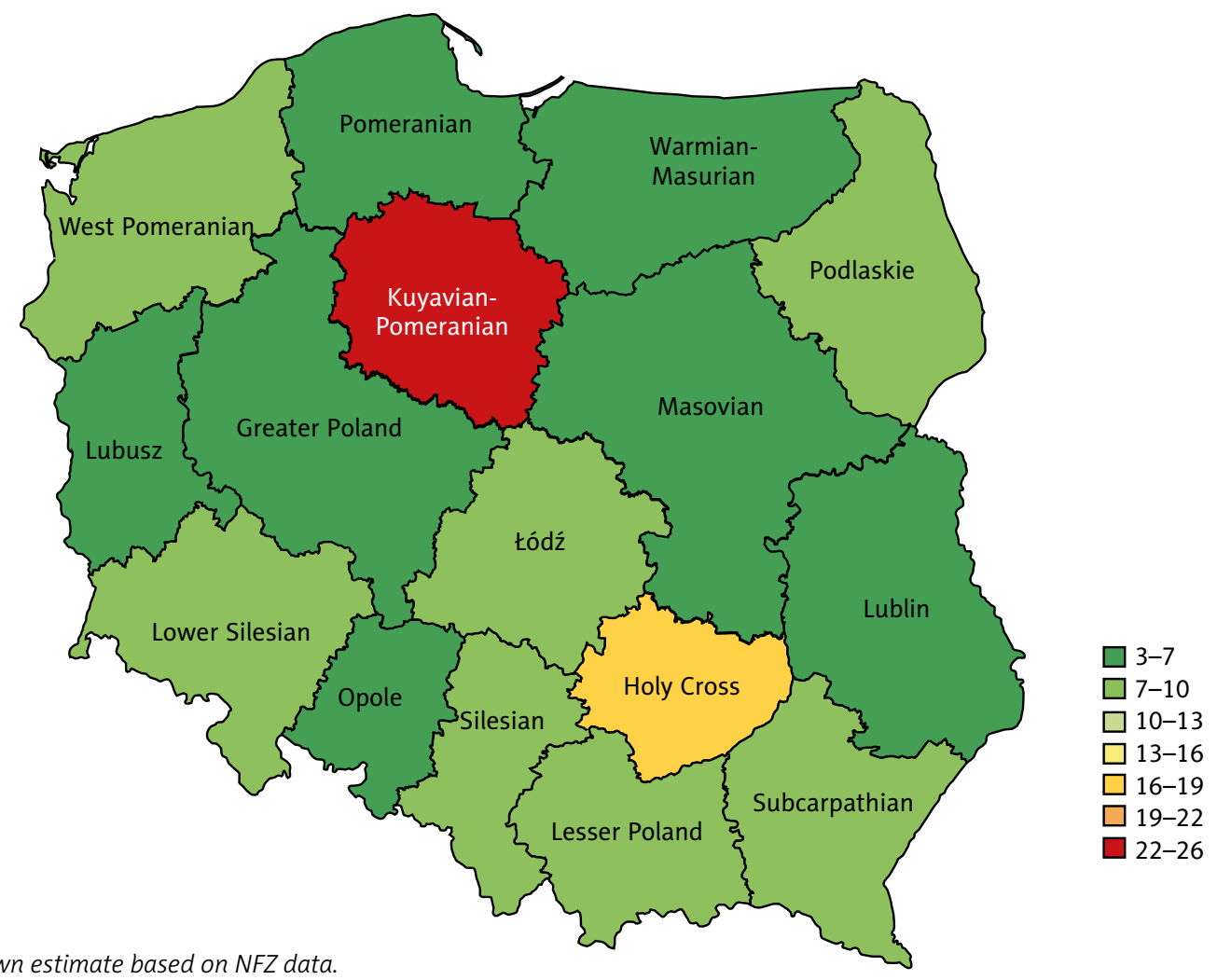

Figure 4. Prevalence of anaphylaxis per 100,000 inhabitants in particular administrative regions (voivodeships) of Poland (2015)

Unspecified anaphylactic shock (T78.2) was the predominant diagnosis (76.5\% of all patients surveyed) in 2015. The next most common diagnosis (16.9\% of patients) was anaphylactic reaction due to food (T78.0), then anaphylactic reaction due to an adverse effect of correct medicinal substance properly administered (T88.6) (8.1\% of patients). According to the NFZ data for 2015, only 10 (0.3\%) patients had an anaphylactic reaction due to serum.
In 2015, the prevalence rate of anaphylaxis according to NFZ data was 8.2/100,000 (8.4/100,000 for females and 7.9/100,000 for males). The highest prevalence rate, $14.5 / 100,000$, was found in women aged 50-54 years. The details are given in Figure 3. The prevalence rate in urban areas was 13.1/100,000 (12.9/100,000 for males and 13.2/100,000 in females) and in rural areas 0.8/100,000 (0.6/100,000 for males and 0.9/100,000 for females). 
Table 1. NFZ expenditure on medical services related to anaphylaxis (2015)

\begin{tabular}{lcccccccc}
\hline Diagnosis & \multicolumn{1}{c}{ Year } \\
\cline { 2 - 8 } & 2008 & 2009 & 2010 & 2011 & 2012 & 2013 & 2014 & 2015 \\
\hline T78.0 & 124831 & 162832 & 132208 & 137035 & 167791 & 146122 & 255426 & 260955 \\
\hline T78.2 & 2990115 & 4494895 & 3249459 & 3220171 & 3564077 & 2943270 & 3051553 & 2715638 \\
\hline T80.5 & 1980 & 13445 & 1356 & 1049 & 656 & 311 & 587 & 5818 \\
\hline T88.6 & 265834 & 366599 & 380673 & 354857 & 246509 & 281021 & 252911 & 510663 \\
\hline Total (PLN) & 3382759 & 5037772 & 3763695 & 3713112 & 3979033 & 3370725 & 3560477 & 3493073 \\
\hline Total (USD) & 1404101 & 1616640 & 1248034 & 1252990 & 1221687 & 1066415 & 1128483 & 926520 \\
\hline Total (EUR) & 961940 & 1164184 & 942196 & 901284 & 950784 & 803032 & 850730 & 834884 \\
\hline
\end{tabular}

Source: Own estimate based on NFZ data.

The prevalence rates of anaphylaxis estimated from the NFZ data demonstrate a clear regional variation. In 2015, the highest prevalences (per 100,000 people) were found in two regions (voivodeships), Kuyavian-Pomeranian at 25.3 and Subcarpathian at 17.2, with the lowest in the Lubusz, Masovian and Warmian-Masurian regions at 3.5, 4.6 and 4.6 respectively. These regional variations are presented in Figure 4. Analysis of linear relationships (Pearson's correlation) demonstrated a weak relationship $(r=0.287)$ between the number of allergists per 100,000 inhabitants in a given administrative region and the estimated prevalence rate of anaphylaxis.

In 2015, of those patients who received healthcare services for anaphylaxis (T78.0, T78.2, T80.5, T88.6), nearly half (46.5\%) were treated in a hospital setting. Another $29.9 \%$ received treatment on an outpatient basis (specialist outpatient care) and $13.5 \%$ were treated in primary care. Emergency medical services provided treatment to the remaining patients (10.1\%).

A total of 10,131 healthcare services for anaphylaxis were provided to the 3144 patients. Of those, $71.8 \%$ were provided on an inpatient basis in a hospital, $19.8 \%$ on an outpatient basis, $4.9 \%$ in primary care and $3.5 \%$ by emergency medical services.

The annual NFZ expenditure on the treatment of anaphylaxis over the period of 2008-2015 remained at PLN 3.4 million to 4 million. The spending was substantially higher in 2009, at PLN 5 million. It should be noted that since the Polish zloty fell against the US dollar and the euro, the NFZ expenditure calculated in USD decreased from USD 1.4 million (EUR 1 million) in 2015 to USD 0.9 million (EUR 0.8 million) in 2015 with simultaneous increases in the number of patients and medical services (Table 1).

\section{Survey}

Of 305 allergists participating in the survey, 73.1\% (95\% Cl: 68.1-78.1\%) treated patients who had experienced an anaphylactic shock $(38.1 \%$ had managed from 1 to 4 such patients, $21.1 \%$ from 5 to 9 patients, $20.2 \%$ from 10 to 19 patients and $20.7 \% 20$ or more of such patients).

Hypersensitivity to insect venom was the most common cause of anaphylaxis in Poland - in 41.4\% (95\% Cl: $40.1-42.8 \%$ ) of patients managed by allergists. Hyper-

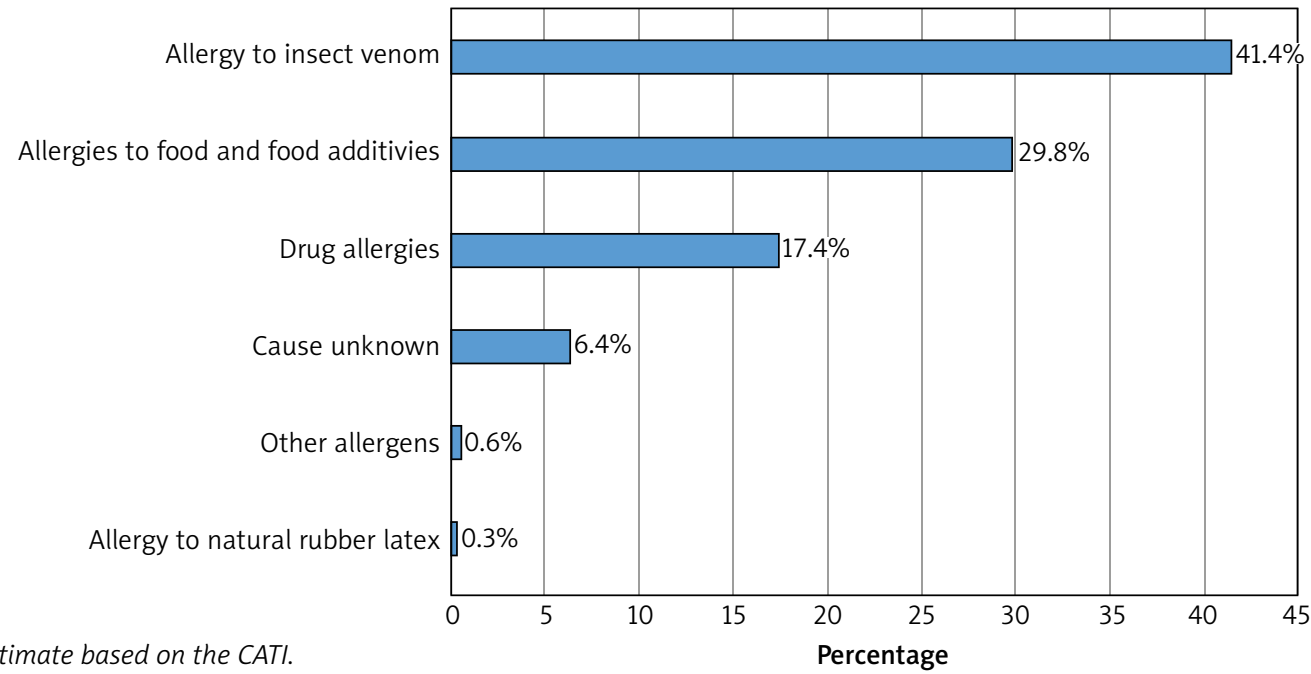

Source: Own estimate based on the CATI.

Percentage

Figure 5 . Causes of anaphylaxis in patients managed by allergists 
sensitivity to food and food additives was the second most common cause of anaphylaxis $(29.8 \%, 95 \% \mathrm{Cl}$ : 28.5-31.1\%) followed by drug allergies (17.4\%, 95\% Cl: 16.4-18.5). The detailed information is given in Figure 5.

Of the allergists managing patients who had experienced anaphylactic shock, 61.9\% (95\% Cl: 55.5-68.3\%) use antihistamines to treat anaphylaxis and $59.2 \%(95 \% \mathrm{Cl}$ : 52.7-65.7\%) use glucocorticoids. All physicians (100\%) declared the use of epinephrine in the cases of anaphylaxis.

\section{Discussion}

In 2013, Panesar et al. carried out a systematic review of the epidemiology of anaphylaxis in Europe. Their findings demonstrated that $0.3 \%$ of the European population experienced anaphylaxis at some point in their lives. The incidence rates for all-causes of anaphylaxis ranged from 1.5 to 7.9 per 100,000 person-years. The annual death rate from anaphylaxis was from $0.000002 \%$ to $0.0001 \%$ [8]. Another systematic review by Tejedor-Alonso et al. from 2015 found substantial variations in the prevalence of anaphylaxis depending on the geographical region (from 20 episodes per 100,000 persons to 5100 per 100,000 ) [6]. The rates calculated in this study seem to be underestimated when compared to the prevalence estimates from other countries. According to our estimates, in 2015 , the prevalence rate of anaphylaxis in Poland was 8.2 per 100,000 versus 30 reported by Panesar et al. for the European population [8] and a similar rate by Tejedor-Alonso et al. [6]. It should be noted, however, that both our estimates and those of Panesar et al. included people who had experienced anaphylaxis at some point in their lives.

The most common causes of anaphylaxis in Poland as described by the allergists surveyed were consistent with the observations by Worm et al. from Germany, Austria and Switzerland, for 2010-2011. In their analyses they demonstrated that insect venom was the most common trigger of anaphylaxis ( $n=2074 ; 50.1 \%$ ), followed by food ( $n=1039 ; 25.1 \%)$ and drugs $(n=627 ; 15.1 \%$ ) [9]. The Polish allergists surveyed also reported that in over $41 \%$ of their patients anaphylaxis was induced by insect venom, followed by food (29.8\%) and drugs (17.4\%). Another study by Worm et al. covering the period of 2011-2014 and based on the data from 59 centers in 10 European countries, reported that anaphylaxis was mainly caused by food (children and adults $64.9 \%$ and $20.2 \%$, respectively), insect venom (20.2\% and $48.2 \%)$ and less frequently by drugs ( $4.8 \%$ and $22.4 \%$ ) [17]. These findings differ from our estimates and the first observations by Worm et al. from the period of 2010-2011 [9]. It should be noted, however, that in both studies, children and adults were considered as one group while the study of Worm et al. conducted for the period of 2011-2014 [17] estimated the rates separately for children and for adults.

There are few studies which estimate and compare the prevalence of anaphylaxis in rural and urban areas. Of the many studies assessing the epidemiology of anaphylaxis, two only deal with the differences in prevalence depending on the patients' residence, urban vs. rural. The study of Mostmans et al. conducted in Belgium for 2009-2013 [18] confirmed a higher prevalence of anaphylaxis in towns and cities compared to rural communities. In the present study, the prevalence of anaphylaxis was 13.1/100,000 in towns and cities vs. 0.8/100,000 in rural communities. On the other hand, Sheikh and Alves in their analysis of English hospital data for the period of 1991-1995 [19] found a lower incidence of anaphylaxis in urban areas than rural areas, $12 / 100,000$ vs. 32/100,000, respectively.

The difference between the prevalence of anaphylaxis between urban and rural areas estimated from the NFZ data may be also related to fewer instances of patients who experienced anaphylactic shock seeking consultation with an allergist, or the patients being less willing to undergo the required diagnostic tests and treatment.

\section{Limitations of the study}

This study was based on two independent sources of data. The NFZ register was established for accounting purposes, not to support research such as epidemiological studies. The NFZ data reports only those healthcare services that are financed from public funds and does not include information on private healthcare, which would lead to underestimating the actual prevalence rates.

According to the methodology of this study, a person with a history of anaphylaxis was defined as any patient who in a given year received a healthcare service related to the diagnosis, either as the principal or accompanying disorder, and encoded as T78.0, T78.2, T80.5 or T88.6. This is why the group of patients with anaphylaxis also included people with an initial suspicion of anaphylaxis, but ultimately diagnosed with another disorder. This may result in overstating the prevalence rates. On the other hand, a person with a past history of anaphylaxis who does not receive healthcare benefits (services) financed from public funds was not included in the present analysis, which in turn would lead to underestimating the prevalence rates. Accordingly, an additional analysis was performed which showed that from 2008-2015, 7975 people made use of medical services for anaphylaxis at least once, 1696 at least twice and 882 at least three times. This means that most people with anaphylaxis benefited from medical services just once, which suggests that the rates presented in this article should be interpreted as incidence rather than prevalence, although this question cannot be definitively resolved.

There is no separate category for the diagnosis of anaphylaxis in the ICD-10 coding system, so anaphylaxis is encoded in the subcategories T78.0, T782, T80.5 and T88.6. It has been observed that some physicians use just the first three characters of the ICD-10 code in reports submitted to the NFZ. In 2015, the diagnosis of $17.9 \%$ of patients was just encoded T78, $77.3 \%$ just T80 and 
of $56.5 \%$ just T88. Of the total number of patients with such a code there was no specific diagnosis in $20.7 \%$ of reports, which may lead to underestimating the actual prevalence rates.

\section{Strengths of the study}

This paper makes use of data from two independent, complementary sources. The NFZ data are official and cover the entire country. The present study analyzes the information from 2008-2015, i.e. 8 reporting periods.

A CATI survey was conducted in a group of 305 of 1250 active allergists in Poland, both in public and in private healthcare, i.e. nearly a quarter (24.7\%) of all physicians that are specialists in allergology. The distribution of the sample across the country corresponds to the distribution of the population.

\section{Conclusions}

The NFZ data are a valuable source of information on individuals with a history of anaphylaxis, yet its usefulness for epidemiological and research studies is limited. A central anaphylaxis registry should be established in Poland to facilitate long-term in-depth observation. This is the only approach that would enhance collecting a wide range of reliable information on the cases, management and consequences of anaphylaxis. The official data, such as those sourced from the NFZ, should serve to complement the registry data. Another concern is the encoding and reporting of diagnosed anaphylaxis cases to the NFZ.

We have found that most people who have experienced anaphylactic shock do not seek further diagnostic investigation or management (at least, those funded by the NFZ). Such people should also be offered adequate care to prevent new episodes of anaphylaxis in the future, including access to self-injectable epinephrine and education to the patients and their families. Anaphylaxis education programs should also include school teachers and other personnel responsible for child care during the school year and in the summer.

As anaphylaxis is often food-induced, more education in this area is needed, including precautionary labelling of foods for allergen content (potential triggers of anaphylaxis).

\section{Conflict of interest}

The authors declare no conflict of interest.

\section{References}

1. Johansson SGO, Bieber T, Dahl R, et al. Revised nomenclature for allergy for global use: Report of the Nomenclature Review Committee of the World Allergy Organization. J Allergy Clin Immunol 2004; 113: 832-36.

2. Sampson HA, Munoz-Furlong A, Campbell RL, et al. Second Symposium of a definition and management of anaphylaxis:
Summary report - Second National Institute of Allergy and Infectious Diseases/Food Allergy and Anaphylaxis Network Symposium. J Allergy Clin Immunol 2006; 117: 391-7.

3. Lockey RF. Anaphylaxis: Synopsis; http://www.worldallergy. org/professional/allergic_diseases_center/anaphylaxis/anaphylaxissynopsis.php [18.04.2016].

4. Nowak RM, Macias CG. Anaphylaxis on the other front line: perspectives from the emergency department. Am J Med 2014; 127: 34-44.

5. World Allergy Organization (WAO). White Book on Allergy: Update 2013. World Allergy Organization 2013.

6. Tejedor-Alonso MA, Moro-Moro M, Múgica-García MV. Epidemiology of anaphylaxis: contributions from the last 10 years. J Investig Allergol Clin Immunol 2015; 25: 163-75.

7. Tejedor-Alonso MA, Moro-Moro M, Múgica-García MV. Epidemiology of anaphylaxis. Clin Exp Allergy 2015; 45: 1027-39.

8. Panesar SS, Javad S, de Silva D, et al. The epidemiology of anaphylaxis in Europe: a systematic review. Allergy 2013; 68: 1353-61.

9. Worm M, Eckermann O, Dölle S, et al. Triggers and treatment of anaphylaxis: an analysis of 4,000 cases from Germany, Austria and Switzerland. Dtsch Arztebl Int 2014; 111: 367-75.

10. Decker WW, Campbell RL, Manivannan V, et al. The etiology and incidence of anaphylaxis in Rochester, Minnesota: a report from the Rochester Epidemiology Project. J Allergy Clin Immunol 2008; 122: 1161-5.

11. Sheikh A, Hippisley-Cox J, Newton J, et al. Trends in national incidence, lifetime prevalence and adrenaline prescribing for anaphylaxis in England. J R Soc Med 2008; 101: 139-43.

12. Francuzik W, Nassiri M, Babina M, et al. Impact of sex on anaphylaxis severity - data from the Anaphylaxis Registry. J Allergy Clin Immunol 2015; 136: 1425-6.

13. Liebhart J. Anafilaksja. Alergia 2013; 4: 47-51.

14. Muraro A, Halken S, Arshad SH, et al. EAACI Food Allergy and Anaphylaxis Guidelines. Primary prevention of food allergy. Allergy 2014; 69: 590-601.

15. Motala C, Fiocchi A. Cow's milk allergy in children; http:// www.worldallergy.org/professional/allergic_diseases_center/cows_milk_allergy_in_children/ [18.04.2016].

16. Heddle R, David BK. Allergy to insect stings and bites; http:// www.worldallergy.org/professional/allergic_diseases_center/insect_allergy/ [18.04.2016].

17. Worm M, Moneret-Vautrin A, Scherer K, et al. First European data from the network of severe allergic reactions (NORA). Allergy 2014; 69: 1397-404.

18. Mostmans Y, Blykers M, Mols P, et al. Anaphylaxis in an urban Belgian emergency department: epidemiology and aetiology. Acta Clin Belg 2016; 71: 99-106.

19. Sheikh A, Alves B. Age, sex, geographical and socio-economic variations in admissions for anaphylaxis: analysis of four years of English hospital data. Clin Exp Allergy 2001; 31: 1571-6. 